\title{
Circ-FURIN Expression Enhances Osteoblast Differentiation In Dental Pulp Stem Cells Via SOX11 Signaling Pathway Sponging miR-125
}

Fang Ji

Shanghai Red Cross Ninth People's Hospital: Shanghai Jiao Tong University School of Medicine Affiliated Ninth People's Hospital

\section{Yueting Lin}

Shanghai Jiao Tong University School of Medicine Affiliated Ninth People's Hospital

Jing Pan

Shanghai Jiao Tong University School of Medicine Affiliated Ninth People's Hospital

\section{Zhao Yang}

Shanghai Jiao Tong University School of Medicine Affiliated Ninth People's Hospital

\section{Qianhui Ren}

Shanghai Jiao Tong University School of Medicine Affiliated Ninth People's Hospital

\section{Jiubin Quan}

Shanghai Jiao Tong University School of Medicine Affiliated Ninth People's Hospital

\section{Jian Wang}

Shanghai Jiao Tong University School of Medicine Affiliated Ninth People's Hospital

Jiang Tao ( $\sim$ doctor_taojiang@126.com )

Shanghai Jiao Tong University School of Medicine Affiliated Ninth People's Hospital

\section{Research Article}

Keywords: dental pulp stem cells, osteoblast differentiation, circ-FURIN, miR-125, SOX11

Posted Date: August 25th, 2021

DOI: https://doi.org/10.21203/rs.3.rs-773181/v1

License: (c) (i) This work is licensed under a Creative Commons Attribution 4.0 International License. Read Full License 


\section{Abstract}

Background: Many studies have found that circRNA plays a part in osteoblast differentiation. However, its mechanism remains unknown.

Methods: High-throughput sequencing was used to identifield the different expression of circRNA during osteogenic dental pulp stem cells (DPSCs) differentiation. Luciferase report analysis and RT-qPCR were used to clarify the expression and regulation relationship among circ-FURIN, miR-125 and SOX11. The heterotopic bone formation experiment was further used to confirm the osteoblast differentiation of DPSC with different expression of circ-FURIN, miR-125 and SOX11.

Results: Study indicated that circ-FURIN expression remarkably increased during osteoblast differentiation, yet circ-FURIN knockdown suppressed it. Bioinformatics and luciferase results discovered that miR-125 is the downstream target of circ-FURIN. Furthermore, circ-FURIN upregulation decreased miR-125 expression. MiR-125 upregulation restored the promotion effect of circ-FURIN on osteogenic DPSC differentiation. Luciferase report analysis verified that SOX11 is miR-125 downstream target. miR125 overexpression suppressed osteogenic DPSC differentiation through targeting SOX11. SOX11 overexpression restored miR-125 inhibitory effect on osteogenic DPSC differentiation. In vivo experiments with heterotopic bone model suggested that circ-FURIN overexpression has crucial function to enhance heterotopic bone formation.

Conclusions: In summary, circ-FURIN enhances osteoblast DPSC differentiation via the SOX11 signaling pathway by sponging miR-125. These findings suggest a novel therapeutic target for osteoporosis treatment.

\section{Introduction}

Dental pulp stem cells (DPSCs), the rapidly proliferating mesenchymal stem cells (MSCs), are ready to differentiate into neuronal, osteoblastic, hepatocytic, and myocytic lineages. DPSC multilineage capacity suggests that these cells might have a wider therapeutic potential compared to lineage-restricted adult stem cell populations, including MSCs [1, 2]. DPSCs are fairly accessible and prevail in the whole life. Interactions between dental papilla and epithelial cells during tooth formation enhance tooth morphogenesis by stimulating mesenchymal cell subpopulations to differentiate into odontoblasts, which then build primary dentin [3]. DPSCs can be used for stem cell therapies, which provide therapeutic capabilities for corneal, cardiovascular, neurologic, oro-facial, diabetic, renal, hepatic, muscular dystrophy, and autoimmune conditions. DPSCs have been increasingly used in areas of regenerative medicine, tissue engineering, and bone disorder therapies $[4,5]$.

Being an indispensable class of ncRNAs, head-to-tail closed loop structure regarding circular RNAs (circRNAs) from 3' to 5 ' tail causes a pronounced stability compared to traditional linear RNAs [6]. CircRNAs are highly expressed in many human genes. In some cases, circRNAs have a high expression compared to their counterpart homologous linear isoforms [7]. CircRNAs exert post-transcriptional and 
transcriptional regulation upon protein sponges [8, 9], miRNA sponges, and translation [10]. The so-called competing endogenous RNA (ceRNA) theory regarding circRNAs is popular. In circRNA nucleotide sequences, circRNAs include several miRNA binding sites, avoiding binding to the mRNA target genes, which inhibit miRNA functions [11, 12]. Our previous study has found that circRNA124534 promotes osteogenic DPSC differentiation through the miR-496/ $\beta$-catenin pathway modulation [13]. hsa_circ_0026827 enhances DPSC osteoblast differentiation via RUNX1 and Beclin1 signaling pathway sponging miR-188-3p [14]. However, circRNA remains poorly studied.

The present study demonstrated circRNA influence on DPSC osteogenesis. High-throughput sequencing discovered that circ-FURIN expression is increased during osteoblast DPSC differentiation. circ-FURIN expression enhances osteoblast differentiation regarding human DPSCs through SOX11 signaling pathway via sponging miR-125. These results indicate underlying therapeutic approaches for bone and periodontal tissue regeneration as well as reveal novel functions potential osteogenic differentiation.

\section{Materials \& Methods}

\section{Ethics statement}

Animal Care Committee at the Ninth People's Hospital in Shanghai Jiao Tong University School of Medicine (Shanghai, China) approved animal experiments.

\section{Human DPSC isolation and identification}

Cells were isolated from dental pulp. Briefly, we removed tissue and immersed it in a digestive solution ( $4.0 \mathrm{mg} / \mathrm{mL}$ dispase and $3.0 \mathrm{mg} / \mathrm{mL}$ : type I collagenase) for $1 \mathrm{~h}$ at $37^{\circ} \mathrm{C}$. It was filtered using $70-\mu \mathrm{m}$ cell strainers to get an hDPSC suspension. The cells were plated in T25 flasks and cultured them in culture medium DMEM/F12 with fetal bovine serum of $10 \%$ and $1 \%$ penicillin/streptomycin at $37^{\circ} \mathrm{C}$ and $5 \% \mathrm{CO}_{2}$.

For cell phenotypic analysis using surface proteins, we harvested DPSCs using $5 \mathrm{mM}$ ethylenediaminetetraacetic acid (EDTA) in phosphate-buffered saline (PBS). We then incubated cells with FITC-conjugated antibodies against human CD44, CD45, CD34, CD29, CD90, CD73, and CD105. Isotype antibodies that matched would serve as controls (Becton Dickinson, San Jose, CA). We washed cells with cold PBS containing $2 \%$ fetal calf serum. We acquired 1000 labeled cells and analyzed them through an immunofluorescence microscope (Becton Dickinson).

\section{Plasmid transfection and construction}

Overexpressed pcDNA3.1-SOX11 and pcDNA3.1-circ-FURIN plasmids were purchased from GeneChem Co., Ltd. (Shanghai, China). The siRNA against circ-FURIN (si-circ-FURIN), miR-125 mimic, and miR-125 inhibitor were also purchased from GeneChem Co., Ltd. Lipofectamine ${ }^{\circledR} 3000$ (Invitrogen Life Technologies, USA) was used for cell transfections following standard manufacturer instructions. 
Total RNA was retrieved from osteogenic DPSC differentiation after 0 or 14 days through TRIzol reagent (Invitrogen, Carlsbad, CA, USA) by using $~ 3 \mu \mathrm{g}$ of RNA from individual samples with VAHTS Total RNAseq (H/M/R) Library Prep Kit from Illumina (Vazyme Biotech Co., Ltd, Nanjing, China) to erase ribosomal RNA and retain other mRNA's, like non-coding RNAs. RNAs were exposed to $40 \mathrm{U}$ of RNase R (Epicenter) at $37^{\circ} \mathrm{C}$ for $3 \mathrm{~h}$ after TRIzol purification. KAPA Stranded RNA-Seq Library Prep Kits (Roche, Basel, Switzerland), which were subjected to deep sequencing via Illumina HiSeq 4000 at Aksomics, Inc. (Shanghai, China), was used to prepare RNA-seq library

\section{Total RNA isolation and quantitative reverse transcription (RT-q)PCR}

Total RNA was isolated from tumor cells, tissues, and serum through TRIzol reagent (Invitrogen) abiding by procedures. RNA sample purity and concentration were detected using a spectrophotometer via measuring absorbance values at 260, 230, and $280 \mathrm{~nm}$ through NanoDrop ND-1000 (Thermo Fisher Scientific, Wilmington, DE, USA). The OD260/OD280 ratios in $[1.8,2.1]$ and OD260/OD230 ratios $>1.8$ were considered excellent. We calculated relative expression using $2^{-\triangle \Delta C t}$ approach. RT-qPCR amplification was performed via the aftermentioned primers: circ-FURIN: forward: 5'-

GTTAGAGGTTTTAAAGTG-3'; reverse: 5'-CATCCAGCTCCAGAGCACCTGG-3'; osterix (OSX): forward: 5'ACTGCCCCACCCCTTAGACA-3'; reverse: 5'-GAGGTGCACCCCCAAACCAA-3'; SOX11: forward: 5'AGCAAGAAATGCGGCAAGC-3'; reverse: 5'-ATCCAGAAACACGCACTTGAC-3'; runt-related transcription factor 2 (RUNX2): forward: 5'-TGGTTACTGTCATGGCGGGTA-3'; reverse: 5'TCTCAGATCGTTGAACCTTGCTA-3'; osteocalcin (OCN): forward: 5'-AGCCACCGAGACACCATGAGA-3'; reverse: 5'-GGCTGCACCTTTGCTGGACT-3'; alkaline phosphatase (ALP): forward: 5'GAACGTGGTCACCTCCATCCT-3'; reverse: 5'-TCTCGTGGTCACAATGC-3'; miR-125: forward: 5'GGGTCCGAGGTATTCGCACT-3'; reverse: 5'-TCCCTGAGACCCTTTAACCTGTG-3'; U6: forward: 5'CTCGCTTCGGCAGCACA-3'; reverse: 5'-AACGCTTCATTTGCGT-3'; and GAPDH: forward: 5'AATCCCATCACCATCTTCC-3'; reverse: 5'-CATCACGCCACAGTTTCC-3'. We normalized circ-FURIN, ALP, SOX11, OCN, OSX, and RUNX2 expression levels to GAPDH, while we normalized miR-125 expression level to U6.

\section{Dual-luciferase reporter assays}

Binding sites for circ-FURIN and SOX11 3'-UTR, i.e., circ-FURIN-WT, circ-FURIN-Mut, SOX11-3'UTR-WT, and SOX11-3'UTR-Mut, were placed into pGL3 promoter vector (Realgene, Nanjing, China) for dual-luciferase reporter assay. Cells were plated into 24-well plates and transfected with $80 \mathrm{ng}$ plasmid, $50 \mathrm{nM}$ miR-125 mimics, $5 \mathrm{ng}$ Renilla luciferase vector pRL-SV40, along with NC reagents through Lipofectamine 2000 (Invitrogen). Cells were gathered and detected two days followed by transfection through Dual-Luciferase Assay (Promega, Madison, WI, USA) according to protocols. All experiments were independently repeated in triplicate.

\section{ALP staining}


We used NBT/BCIP staining kit (CoWin Biotech, Beijing, China) for ALP staining following instructions. DPSCs were seeded in 24-well plates to be cultured in osteogenic medium for 1 2 w. Cellswere fixed with $4 \%$ PFA for half of an hour and incubated them in a staining reagent in dark for twenty minutes.

\section{Mineralization assay}

In order for detection of extracellular matrix calcium deposition, we seeded DPSCs in 24-well tissue plates and cultivated them for one to two weeks in osteogenic medium. Then, $0.1 \%$ Alizarin Red S (ARS, SigmaAldrich, Saint Louis, MO, USA) solution at $\mathrm{pH} 4.2$ was used to stain nodules that calcified followed by fixing DPSCs.

\section{In vivo heterotopic bone formation assay}

DPSCs were induced in osteogenic medium for one week before in vivo investigation. Cells were resuspended to be incubated with $7 \mathrm{~mm} \times 5 \mathrm{~mm} \times 2 \mathrm{~mm}$ Bio-Oss Collagen (Geistlich, GEWO GmbH, Baden-Baden, Germany) scaffolds for one hour at $37^{\circ} \mathrm{C}$ after centrifugation at $150 \mathrm{~g}$ for 5 min. Cells were subcutaneously implanted on backs of five-week-old BALB/c homozygous nude (nu/nu) mice (five mice per group) as previously described [15]. The implants were harvested after eight weeks.

\section{Hematoxylin and eosin (H\&E) and Masson's trichrome staining and immunohistochemical analyses}

Specimens in 10\% EDTA (pH 7.4) was decalcified for 1 min after embedding in paraffin and dehydration. We cut sections ( $5 \mu \mathrm{m})$ and stained them with H\&E as well as Masson's trichrome. We performed immunohistochemical analysis following previously described procedure [16]. Specimens were maintained in $5 \%$ normal goat serum for half of an hour and incubated with primary antibody against OCN (Santa Cruz Biotechnology) at $4^{\circ} \mathrm{C}$ overnight. Our team processed sections via ABC detection kit (Vector Laboratories, Burlingame, CA) and visualized with Olympus microscope (Olympus Co., Tokyo, Japan).

\section{Statistical analyses}

Our team expressed results as mean \pm standard deviation (SD). GraphPad Prism software (GraphPad, La Jolla, CA, USA) was used to compute significance. $P$-value $\leq 0.05$ inferred statistical significance.

\section{Results}

\section{DPSC circ-FURIN expression increases during osteogenic differentiation}

Isolated DPSCs have a classic cobblestone-like morphology (Figure. 1A). Immunofluorescence staining demonstrated that isolated DPSCs were negative for CD45 and CD34 expression, yet positive for mesenchymal cell surface markers CD44, CD29, CD90, CD73, and CD105 (Figure. 1B-H). Osteogenic DPSC potential was analyzed using ARS and ALP staining. The result illustrated that odontogenic induction promoted osteogenic DPSC differentiation (Figure. 2A). RT-qPCR verified that mRNA expression 
concerning osteogenic markers $O C N, A L P, R U N X 2$, and $O S X$ consistently remarkably increased during osteogenic differentiation (Figure. 2B). Previous research has discovered that abnormal circRNA expression correlates with osteogenic differentiation $[14,17]$. The present study employed nextgeneration sequencing to detect abnormal circRNA expression during osteogenic differentiation in DPSCs. The results showed that circRNA was abnormally expressed (Figure. 2C). RT-qPCR analysis showed that hsa_circ_0036872, hsa_circ_00056856, hsa_circ_0033144, hsa_circ_0002003, hsa_circ_0008615, and hsa_circ_0006127 had different expressions. Furthermore, hsa_circ_0036872 expression enhanced significantly after osteogenic differentiation induction for 14 day when compared to normal DPSCs (Figure. 2D).

\section{Overexpression of circ-FURIN promotes osteogenic DPSC differentiation}

The recent research determined that hsa_circ_0036872 was cyclized and derived from FURIN gene exon that is located at chr15:91411884-91426687. Therefore, hsa_circ_0036872 was named circ-FURIN (Figure. 3A). RT-qPCR analysis illustrated that circ-FURIN expression increased significantly after transfection regarding circ-FURIN overexpression vector. Circ-FURIN silencing significantly suppressed circ-FURIN expression (Figure. 3B).

The ARS and ALP detection of osteogenic DPSC differentiation after two weeks of induction showed that circ-FURIN overexpression promoted osteogenic differentiation, while circ-FURIN silencing decreased it (Figure. 3C). RT-qPCR results showed that circ-FURIN overexpression promoted RUNX2, OCN, ALP, and OSX expression, while circ-FURIN silencing decreased it (Figure. 3D-G).

\section{MiR-125 and SOX11 are circ-FURIN downstream targets}

Venn diagrams were created using the interactome, starBank, and circBank websites for predicting miRNAs sponged by circ-FURIN. The results showed that 43 miRNAs can interact with circ-FURIN (Figure. 4A). Dual-luciferase reporter assays validated that only miR-125 markedly reduced luciferase activity in HEK293T cells during co-transfection with WT and different miRNA mimics (Figure. 4B). This suggests that miR-125 is circ-FURIN downstream target. To uncover interaction correlation between circ-FURIN and miR-125, we expected binding sites between miR-125 and circ-FURIN using starbase. A luciferase vector with or without a mutation was then constructed (Figure. 4C), combined with miR-125 mimics, and transfected into DPSCs. Luciferase reporter analyses verified that miR-125 inhibited the luciferase activity in circ-FURIN WT cells and did not affect activity in mutated cell lines, indicating that miR-125 is a potential circ-FURIN target (Figure. 4D).

Bioinformatics analysis advised that SOX11 is an miR-125 target and that miR-125 directly interacts with SOX11 3'-UTR to suppress mRNA expression (Figure. 4E). MiR-125 also inhibited luciferase activity in WT and yet in Mut cell lines (Figure. 4F). Integrated data revealed that circ-FURIN overexpression promotes osteogenic DPSC differentiation by regulating the miR-125/SOX11 axis. 


\section{MiR-125 overexpression or SOX11 downregulation restores circ-FURIN promotion effect on osteogenic DPSC differentiation}

To characterize the regulatory relationship among circ-FURIN, miR-125, and SOX11, DPSCs were transfected with circ-FURIN overexpression, miR-125 mimic, or SOX11 silencing (si-SOX11) vectors either separately or together. RT-qPCR analyses confirmed that circ-FURIN expression significantly increased after circ-FURIN overexpression vector transfection. MiR-125 overexpression or SOX11 silencing seldom reversed the circ-FURIN expression (Figure. 5A). Circ-FURIN overexpression decreased miR-125 expression in DPSCs. Cells treated with miR-125 mimic promoted miR-125 expression by silencing SOX 11 but did not reverse miR-125 expression (Figure. 5B). In addition, circ-FURIN overexpression increased SOX11 expression in myoblast cells, while miR-125 mimic treatment partially suppressed SOX11 expression. Transfection with SOX11 silencing vector decreased SOX11 expression (Figure. 5C). These results indicated that miR-125 and SOX11 are circ-FURIN downstream targets, while SOX11 is miR-125 downstream target.

ALP and ARS detection results for osteogenic DPSC differentiation after 14 days of induction showed that miR-125 overexpression or SOX11 downregulation restored the promotion effect of circ-FURIN on osteogenic DPSC differentiation (Figure. 5D). RT-qPCR analysis showed that miR-125 overexpression or SOX11 downregulation restored the promotion effect of circ-FURIN on RUNX2, OCN, ALP, and OSX expression during osteogenic differentiation (Figure. $5 \mathrm{E}-\mathrm{H}$ ).

\section{SOX11 overexpression restores miR-125 inhibitory effect on osteogenic DPSC differentiation}

RT-qPCR detection suggested that miR-125 expression is significantly increased after miR-125 mimics transfection. Transfection with SOX11 overexpression vector had no effect on miR-125 expression (Figure. 6A). RT-qPCR analysis suggested that miR-125 overexpression reduced SOX11 expression. However, treatment with SOX11 overexpression vector significantly promoted SOX11 expression (Figure. 6B). ALP and ARS results after 14 days of induction showed that SOX11 overexpression restored miR-125 inhibitory effects on osteogenic DPSC differentiation (Figure. 6C). RT-qPCR analysis suggested that SOX11 overexpression restored the inhibitory influence of miR-125 on RUNX2, OCN, ALP, and OSX expression during osteogenic differentiation (Figure. 6D-G).

\section{Circ-FURIN expression plays an important role to promote heterotopic bone formation in vivo}

To clarify if circ-FURIN expression influences bone formation in vivo, DPSCs expressing si-circ-FURIN, circFURIN, and negative control (NC) samples were loaded on Bio-Oss collagen scaffolds and implanted them into subcutaneous tissue in nude mice (five mice per group; Figure. 7A). Implanted samples were harvested and studied after eight weeks. H\&E staining levels for bone tissue and Masson's trichrome staining levels for collagen organization were remarkably higher in implants containing circ-FURIN overexpression cells and not thicker in si-circ-FURIN group. In addition, osteoblasts and bone trabeculae were positive regarding $\mathrm{OCN}$, which was detected via immunohistochemical staining. Staining intensity 
and size increased in circ-FURIN overexpression group, while decreased in circ-FURIN downregulation group (Figure. 7).

\section{Discussion}

Research have discovered that circRNA plays an indispensable role during osteogenic differentiation [18, 19]. The present study isolated DPSCs and found that circRNA was abnormally expressed during osteogenesis differentiation using high-throughput sequencing. Study results also verified that circ-FURIN expression was increased during osteogenesis differentiation. Overexpressing circ-FURIN promoted osteogenesis DPSC differentiation, while downregulating circ-FURIN suppressed it. To determine the regulatory mechanism of circ-FURIN in osteogenesis differentiation, bioinformatics analysis and luciferase reporting experiments confirmed that miR-125 is the target of circ-FURIN.

MiR-125 is broadly expressed in various cells and tissues. It binds disparate proteins to inhibit cancer occurrence and is involved in immunity, heart protection from ischemic injury, differentiation of skeletal muscle cells, and angiogenesis inhibition [20-24]. Previous studies have also found that miR-125 promotes osteoclastogenesis [25], suggesting that miR-125 expression can inhibit osteogenic differentiation. It was also discovered that miR-125 expression decreases during osteogenic differentiation, circ-FURIN overexpression suppresses miR-125 expression, while miR-125 overexpression restores the promotion effect of circ-FURIN on osteogenic DPSC differentiation.

Further research advised that SOX11 is the downstream target of miR-125, which was confirmed by luciferase report analysis. Sox genes encode transcription factors containing high mobility group interacting with DNA binding domains. Sox proteins were subgrouped into 8 different divisions (SoxASoxH) based upon their protein specificity $[26,27]$. SOX11 expression was increased in mesenchymal cells stimulating osteoblast differentiation [28]. The present study found that circ-FURIN overexpression promotes SOX11 expression, SOX11 downregulation restores the promotion effect of circ-FURIN on osteogenic DPSC differentiation, while SOX11 overexpression restores miR-125 inhibitory effect upon osteogenic DPSC differentiation. This suggests that circ-FURIN expression promotes osteoblast differentiation of human DPSCs via SOX11 signaling pathway by sponging miR-125.

\section{Conclusions}

Present study showed that abnormal circ-FURIN expression correlates with osteogenic DPSC differentiation. Circ-FURIN also promotes human DPSCs osteoblast differentiation through SOX11 signaling pathway sponging miR-125. These findings do not only add to the body of knowledge on circFURIN role in osteogenic differentiation, but also provide new insight into bone regeneration treatment.

\section{Abbreviations}


DPSCs, dental pulp stem cells; MSCs, mesenchymal stem cells; circRNAs, circular RNAs; ceRNA, competing endogenous RNA; FBS, fetal bovine serum; ALP, alkaline phosphatase.

\section{Declarations}

\section{Ethics approval and consent to participate}

Animal Care Committee at the Ninth People's Hospital in Shanghai Jiao Tong University School of Medicine (Shanghai, China) approved animal experiments.

\section{Consent for publication}

Not applicable.

\section{Availability of data and material}

All data in this study was availability.

\section{Funding}

This work was supported by grants from STCSM (20ZR1431300, 18ZR1422700).

\section{Acknowledgements}

None.

\section{Author Contributions}

F.J.: conception, data gathering, manuscript drafting; Y.L.: design and data collection, interpretation and analyses; J.P.: data interpretation and analysis; Z.Y.: data collection and analysis; Q.R.: study material provision; J.Q.: data collection and assembly; J.W.: data interpretation and collection; J.T.: study material provision, data gathering, financial support, manuscript writing. All authors have read and approved the manuscript.

\section{Conflicts of Interest}

None.

\section{References}

1. Ferro F, Spelat R, Baheney CS. Dental pulp stem cell (DPSC) isolation, characterization, and differentiation. Methods Mol Biol. 2014;1210:91-115.

2. Nuti N, Corallo C, Chan BM, et al. Multipotent Differentiation of Human Dental Pulp Stem Cells: a Literature Review. Stem Cell Rev Rep. 2016;12:511-23. 
3. Gronthos S, Mankani M, Brahim J, et al. Postnatal human dental pulp stem cells (DPSCs) in vitro and in vivo. Proc Natl Acad Sci U S A. 2000;97:13625-30.

4. Botelho J, Cavacas MA, Machado V, et al. Dental stem cells: recent progresses in tissue engineering and regenerative medicine. Ann Med. 2017;49:644-51.

5. Ferrarotti F, Romano F, Gamba MN, et al. Human intrabony defect regeneration with micrografts containing dental pulp stem cells: A randomized controlled clinical trial. J Clin Periodontol. 2018;45:841-50.

6. Li X, Zheng Y, Huang Y, et al. Circular RNA CDR1as regulates osteoblastic differentiation of periodontal ligament stem cells via the miR-7/GDF5/SMAD and p38 MAPK signaling pathway. Stem Cell Res Ther. 2018;9:232.

7. Barrett SP, Salzman J. Circular RNAs: analysis, expression and potential functions. Development. 2016;143:1838-47.

8. Qu S, Zhong Y, Shang R, et al. The emerging landscape of circular RNA in life processes. RNA Biol. 2017;14:992-9.

9. Qu S, Yang X, Li X, et al. Circular RNA: A new star of noncoding RNAs. Cancer Lett. 2015;365:141-8.

10. Yang Y, Fan X, Mao M, et al. Extensive translation of circular RNAs driven by N(6)-methyladenosine. Cell Res. 2017;27:626-41.

11. Hansen TB, Jensen $\mathrm{TI}$, Clausen $\mathrm{BH}$, et al. Natural RNA circles function as efficient microRNA sponges. Nature. 2013;495:384-8.

12. Wang K, Gan TY, Li N, et al. Circular RNA mediates cardiomyocyte death via miRNA-dependent upregulation of MTP18 expression. Cell Death Differ. 2017;24:1111-20.

13. Ji F, Pan J, Shen Z, et al. The Circular RNA circRNA124534 Promotes Osteogenic Differentiation of Human Dental Pulp Stem Cells Through Modulation of the miR-496/beta-Catenin Pathway. Front Cell Dev Biol. 2020;8:230.

14. Ji F, Zhu L, Pan J, et al. hsa_circ_0026827 Promotes Osteoblast Differentiation of Human Dental Pulp Stem Cells Through the Beclin1 and RUNX1 Signaling Pathways by Sponging miR-188-3p. Front Cell Dev Biol. 2020;8:470.

15. Jin C, Jia L, Huang Y, et al. Inhibition of IncRNA MIR31HG Promotes Osteogenic Differentiation of Human Adipose-Derived Stem Cells. Stem Cells. 2016;34:2707-20.

16. Wei J, Li H, Wang S, et al. let-7 enhances osteogenesis and bone formation while repressing adipogenesis of human stromal/mesenchymal stem cells by regulating HMGA2. Stem Cells Dev. 2014;23:1452-63.

17. Ouyang Z, Tan T, Zhang X, et al. CircRNA hsa_circ_0074834 promotes the osteogenesis-angiogenesis coupling process in bone mesenchymal stem cells (BMSCs) by acting as a ceRNA for miR-942-5p. Cell Death Dis. 2019;10:932.

18. Han S, Kuang M, Sun C, et al. Circular RNA hsa_circ_0076690 acts as a prognostic biomarker in osteoporosis and regulates osteogenic differentiation of hBMSCs via sponging miR-152. Aging 
(Albany NY). 2020;12:15011-15020.

19. Yang L, Zeng Z, Kang N, et al. Circ-VANGL1 promotes the progression of osteoporosis by absorbing miRNA-217 to regulate RUNX2 expression. Eur Rev Med Pharmacol Sci. 2019;23:949-57.

20. Shaham L, Binder V, Gefen N, et al. MiR-125 in normal and malignant hematopoiesis. Leukemia. 2012;26:2011-8.

21. Wang JK, Wang Z, Li G. MicroRNA-125 in immunity and cancer. Cancer Lett. 2019;454:134-45.

22. Lozano-Velasco E, Galiano-Torres J, Jodar-Garcia A, et al. miR-27 and miR-125 Distinctly Regulate Muscle-Enriched Transcription Factors in Cardiac and Skeletal Myocytes. Biomed Res Int. 2015;2015:391306.

23. Shenoy A, Danial M, Blelloch RH. Let-7 and miR-125 cooperate to prime progenitors for astrogliogenesis. EMBO J. 2015;34:1180-94.

24. Liu J, Guo B, Chen Z, et al. miR-125b promotes MLL-AF9-driven murine acute myeloid leukemia involving a VEGFA-mediated non-cell-intrinsic mechanism. Blood. 2017;129:1491-502.

25. Sun L, Lian JX, Meng S. MiR-125a-5p promotes osteoclastogenesis by targeting TNFRSF1B. Cell Mol Biol Lett. 2019;24:23.

26. Lefebvre V, Dumitriu B, Penzo-Mendez A, et al. Control of cell fate and differentiation by Sry-related high-mobility-group box (Sox) transcription factors. Int J Biochem Cell Biol. 2007;39:2195-214.

27. Koopman P, Schepers G, Brenner S, et al. Origin and diversity of the SOX transcription factor gene family: genome-wide analysis in Fugu rubripes. Gene. 2004;328:177-86.

28. Gadi J, Jung SH, Lee MJ, et al. The transcription factor protein Sox11 enhances early osteoblast differentiation by facilitating proliferation and the survival of mesenchymal and osteoblast progenitors. J Biol Chem. 2013;288:25400-13.

\section{Figures}
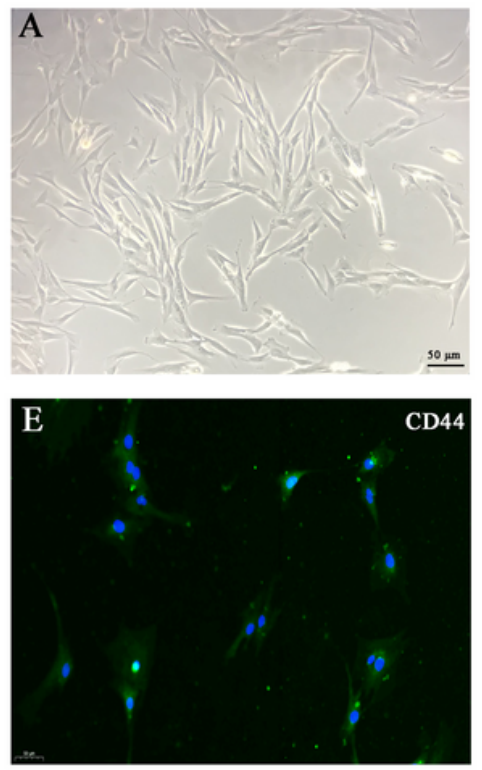
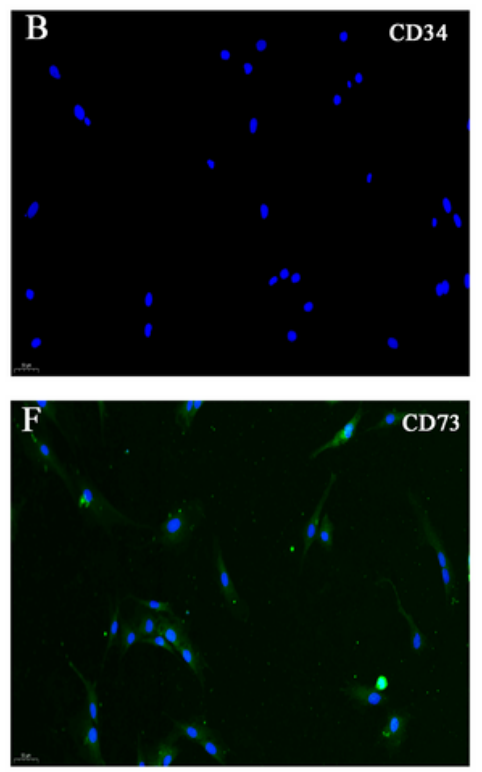
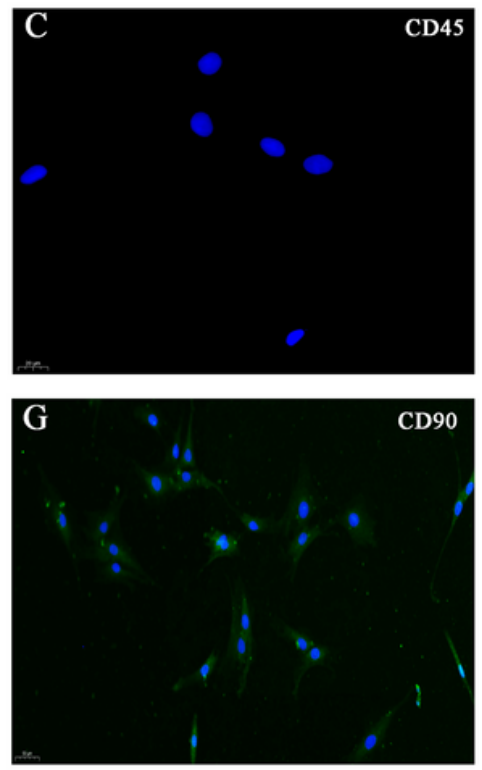
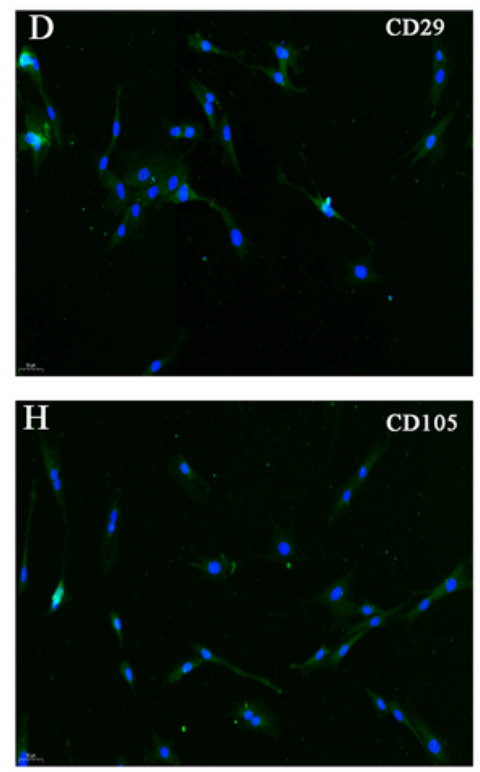
Figure 1

DPSC isolation and characterization. (A) DPSCs adopt fibroblast-like morphology. (B-H) DPSC surface protein profiles analyzed using immunofluorescence.

A
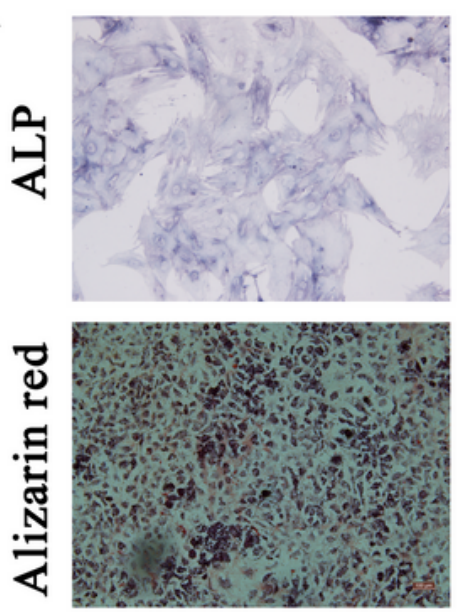

$\mathrm{C}$
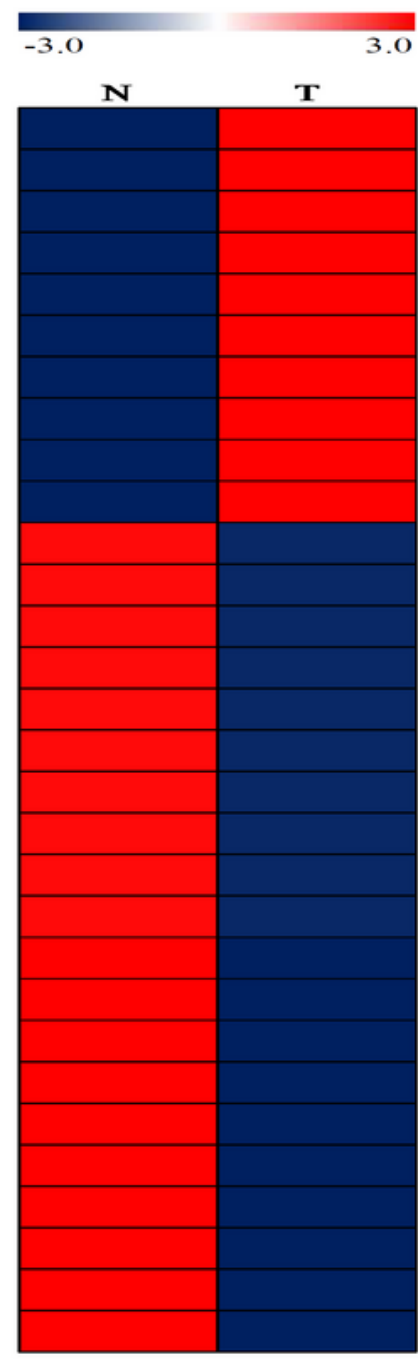

hsa_circ_0036872

hsa_circ_oos 6856

hsa_circ_0033144

hsa_circ_0002003

hsa_circ_ooo8615

hsa_circ_0006127

hsa_circ_oOO3456

hsa_circ_0004115

hsa_circ_0000067

hsa_circ_oO50119

hsa_circ_o006796

hsa_circ_oO15844

hsa_circ_oO12186

hsa_circ_oOO5371

hsa_circ_OOOO213

hsa_circ_0027379

hsa_circ_0003784

hsa_circ_ooo 8488

hsa_circ_0035269

hsa_circ_ooo 4374

hsa_circ_0004709

hsa_circ_ooo 8075

hsa_circ_ooo 8934

hsa_circ_0075796

hsa_circ_OOO2799

hsa_circ_oooo852

hsa_circ_0002599

hsa_circ_oOOO119

hsa circ 0008996

hsa_circ_ooo8922
B

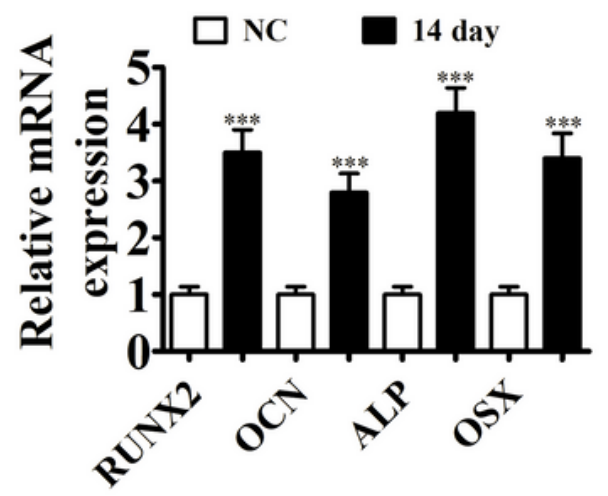

D

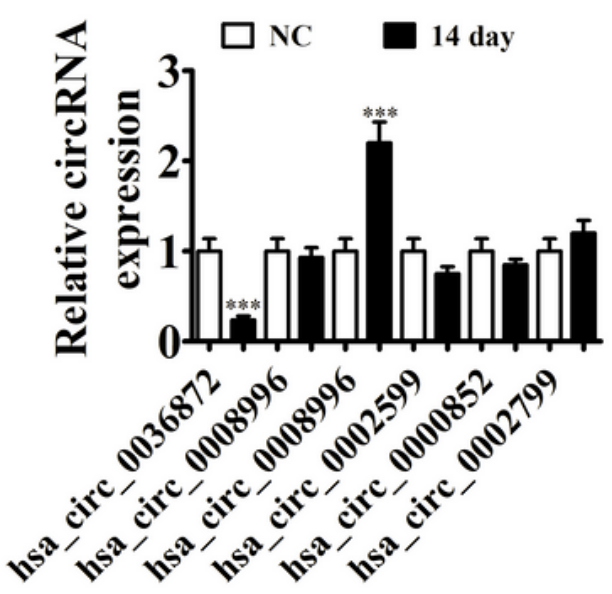

Figure 2 
Circ-FURIN expression increases via osteogenic DPSC differentiation. (A) ALP images and Alizarin Red staining show osteogenic DPSC differentiation potential. (B) RUNX2, OCN, ALP, and OSX expression analysis using RT-qPCR. Results are presented as mean \pm SD. ${ }^{\star} * * P<0.001$ vs. control ( 0 day). (C) Heat map from circRNA high-throughput sequencing data shows differentially expressed circRNA (fold $>3$ ). (D) RT-qPCR detection shows expression of six upregulation circRNAs (including hsa_circ_0036872, hsa_circ_00056856, hsa_circ_0033144, hsa_circ_0002003, hsa_circ_0008615, and hsa_circ_0006127). Results are presented as mean $\pm \mathrm{SD}$. ${ }^{* *} \mathrm{P}<0.001$ vs. control ( 0 day).

A

\section{chr15:91411884-91426687}

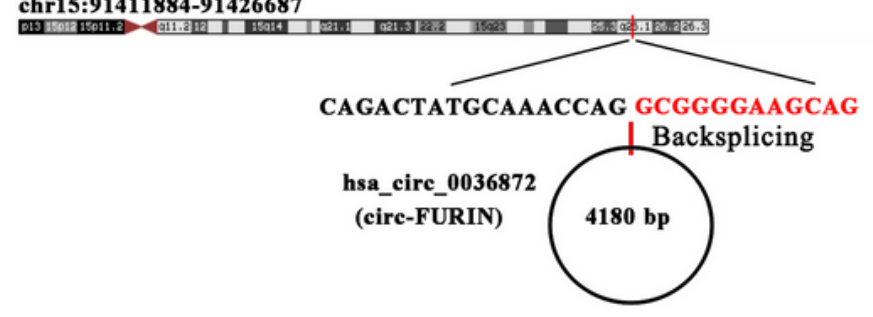

$\mathbf{C}$

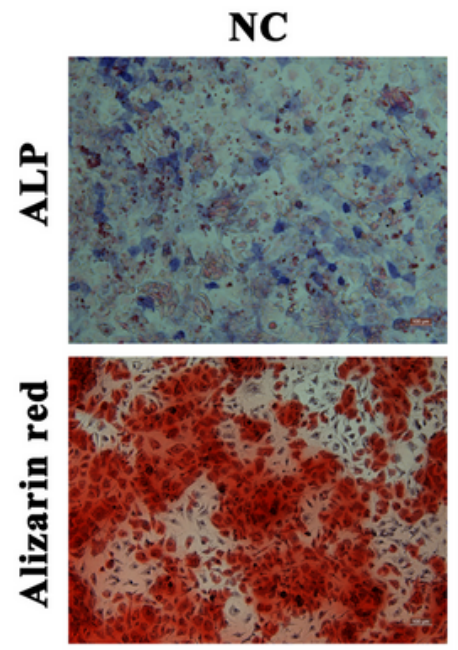

D

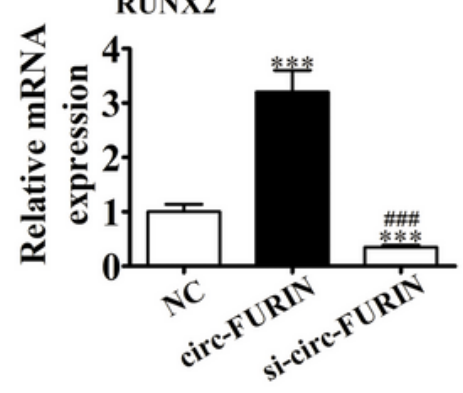

$\mathbf{F}$

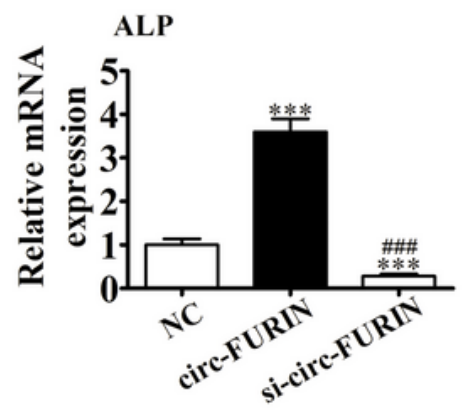

B

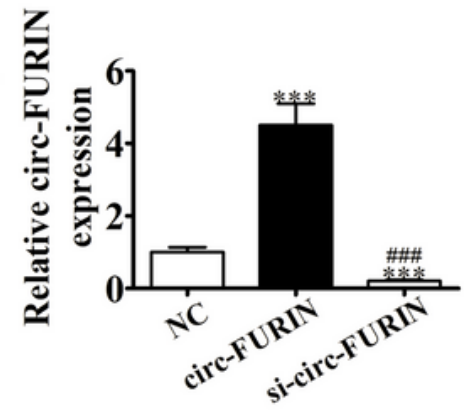

circ-FURIN

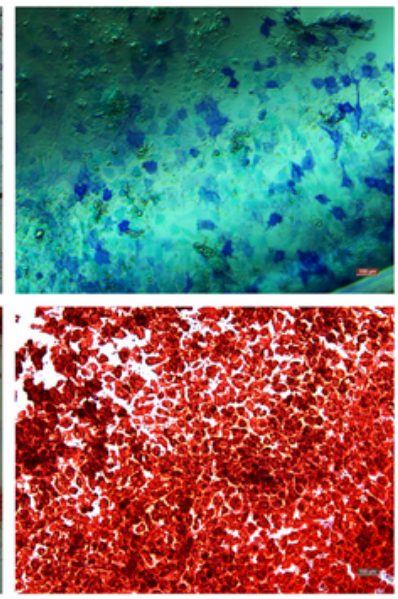

E
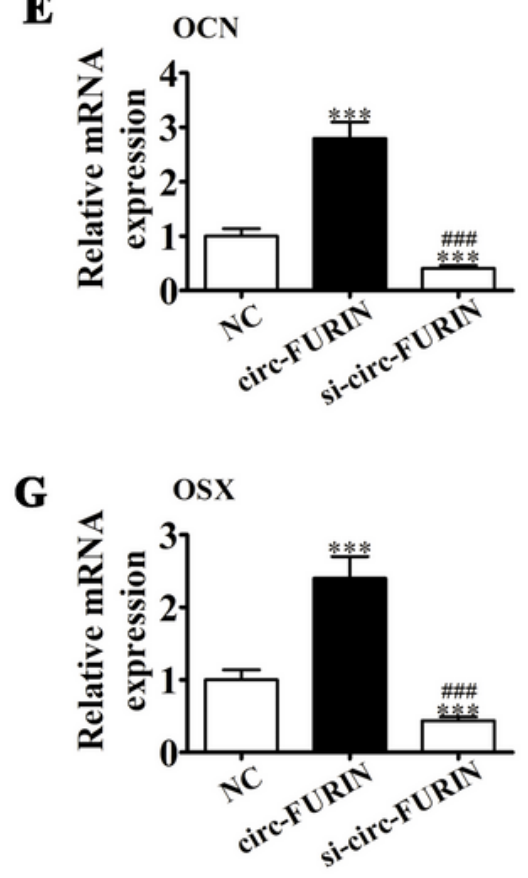


\section{Figure 3}

Circ-FURIN overexpression promotes osteogenic DPSC differentiation. (A) Genomic loci of FURIN gene and hsa_circ_0036872. Red sign indicates back-splicing. (B) RT-qPCR detection shows expression of circFURIN in DPSCs after transfection with circ-FURIN overexpression vector or siRNA against circ-FURIN.

Results are presented as mean \pm SD. $* \star \star P<0.001$ vs. NC. \#\#\#P<0.001 vs. circ-FURIN. (C) ALP images and Alizarin Red staining show osteogenic DPSC differentiation potential after induction for 14 days. (D-G) RT-qPCR shows expression of RUNX2, OCN, ALP, and OSX. Results are presented as mean \pm SD. $\star * * P<0.001$ vs. NC. \#\#\#P<0.001 vs. circ-FURIN.

A

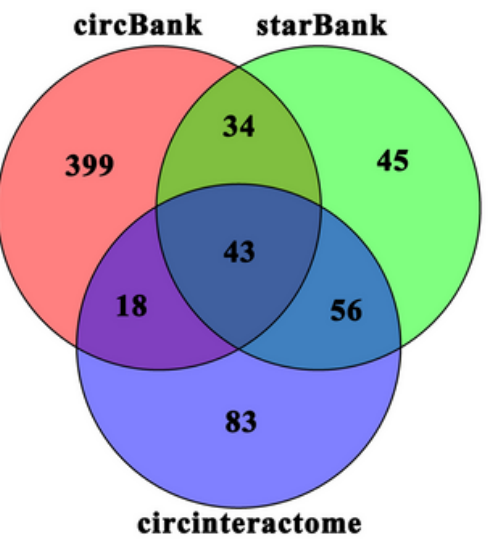

D
circ-FURIN-WT $\quad 5^{\prime}$ uccccuGUGGGGAUCUCAGGGg $3^{\prime}$ miR-125 3' aguguuCAAUCCCAGAGUCCCu 5 circ-FURIN-Mut $\quad 5^{\prime}$ uccccuGUGGGGAUCUAGCCCg $3^{\prime}$

B

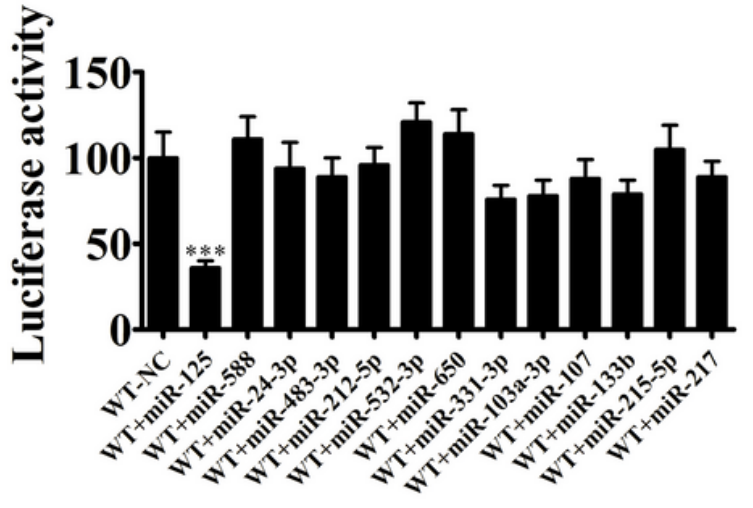

C

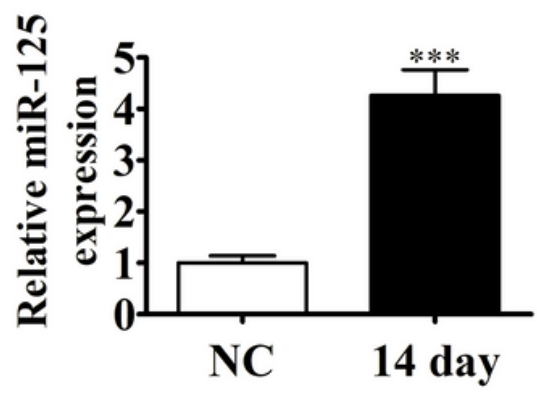

$\mathbf{F}$

SOX11 3' UTR-WT $5^{\prime}$...UAUGCAGUGUUAAUG--CUCAGGGU... 3' miR-125 $3^{\prime} \quad$ AGUGUUCAAUCCCAGAGUCCCU $5^{\prime}$

SOX11 3' UTR-Mut $\quad 5^{\prime} \quad$...UAUGCAGUGUUAAUG--CUAGCCCU... $3^{\prime}$

\section{$\mathbf{E}$}
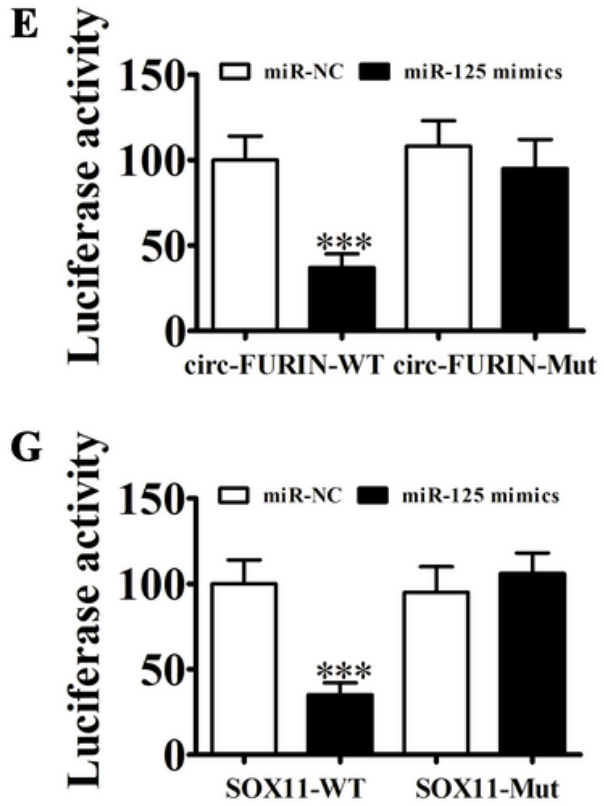

Figure 4

MiR-125 and SOX11 are downstream targets of circ-FURIN. (A) Venn diagrams from interactome, starBank, and circBank websites for predicting miRNAs sponged by circ-FURIN. (B) Dual-luciferase reporter assays show that co-transfection with WT and mimic miR-125 markedly decreases luciferase activity in HEK293T cells. Data are presented as means \pm SD. $* \star \star P<0.001$. (C) RT-qPCR shows miR-125 expression in DPSCs during osteogenic differentiation. Data are presented as means $\pm S D$. ${ }^{\star *} P<0.001$ vs. NC. (D) MiR-125 binding site prediction in circ-FURIN. Mutated version of circ-FURIN is also shown. (E) 
Relative luciferase activity was determined $48 \mathrm{~h}$ after transfection with miR-125 mimic/NC or circ-FURIN wild/Mut in HEK293T cells. Data are presented as means \pm SD. $* \star * P<0.001$. (F) MiR-125 binding site prediction with 3'UTR of SOX11. Mutated version of 3'UTR-SOX11 is also shown. (G) Relative luciferase activity was determined $48 \mathrm{~h}$ after transfection with miR-125 mimic/NC or 3'UTR-SOX11 wild/Mut in HEK293T cells. Data are presented as means \pm SD. *** $P<0.001$.

A

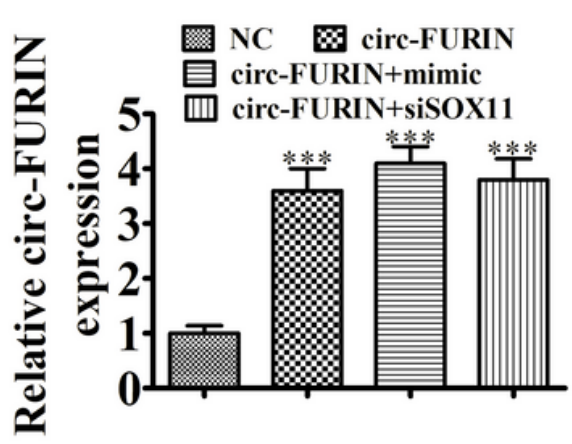

D

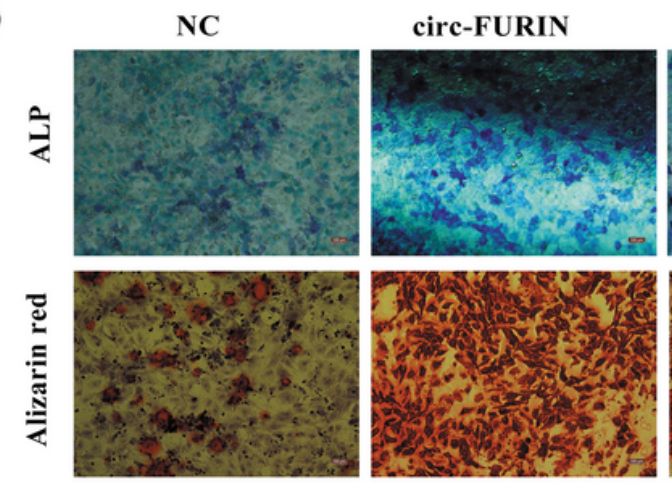

B

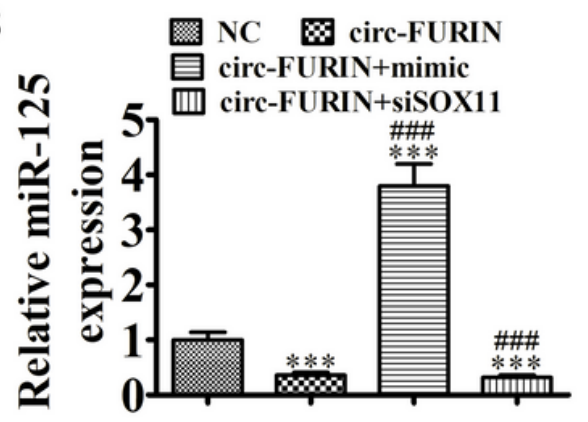

circ-FURIN+mimic circ-FURIN+siSOX11

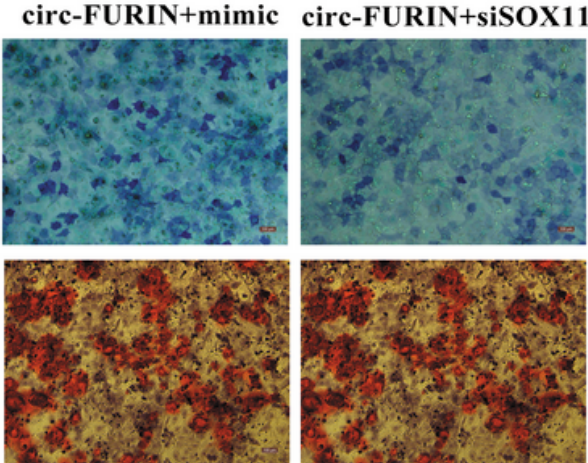

G

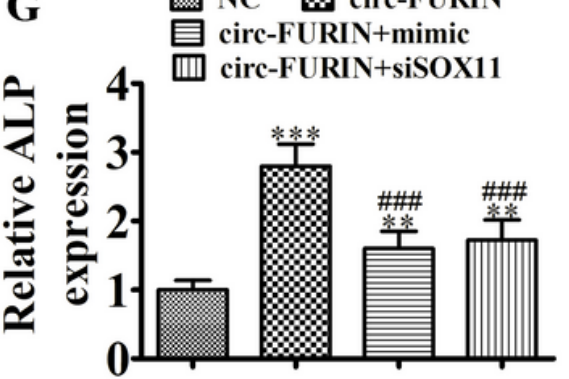

C

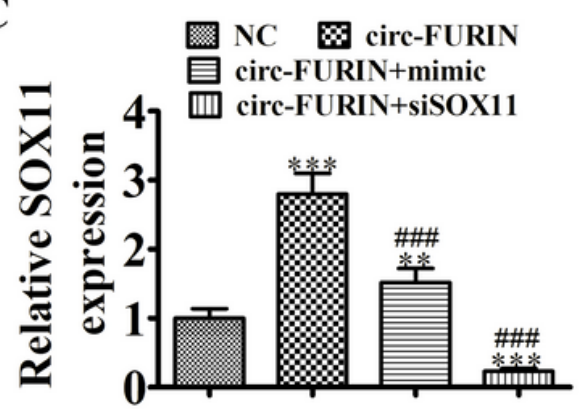

$\mathbf{E}$

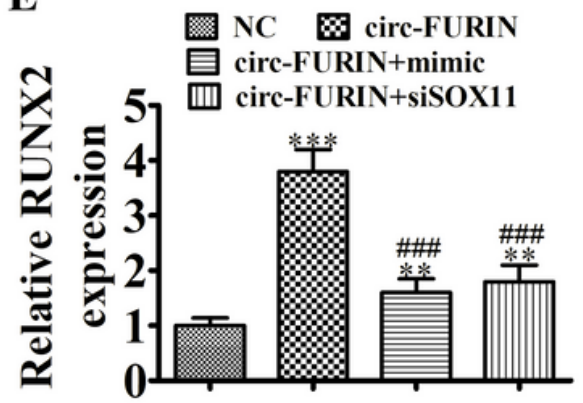

$\mathbf{H}$

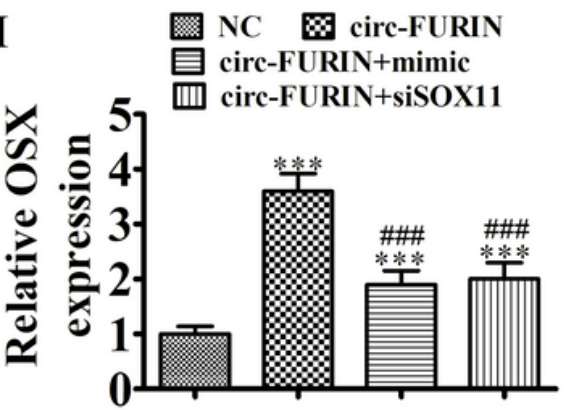

Figure 5

MiR-125 overexpression or SOX11 downregulation restore circ-FURIN promotion effect in osteogenic DPSC differentiation. (A-C) RT-qPCR shows miR-125, SOX11, and circ-FURIN expression. Data are presented as means $\pm S D$. ${ }^{\star \star} P<0.01,{ }^{\star} * \star P<0.001$ vs. NC. \#\#\#P<0.001 vs. circ-FURIN. (D) ALP images and Alizarin Red staining show osteogenic DPSC differentiation potential after induction for 14 days. $(\mathrm{E}-\mathrm{H})$ RT-qPCR shows RUNX2, OCN, ALP, and OSX expression. Results are presented as mean $\pm S D$. ${ }^{\star \star P}<0.01$, $\star \star \star P<0.001$ vs. NC. \#\#\#P<0.001 vs. circ-FURIN. 
A

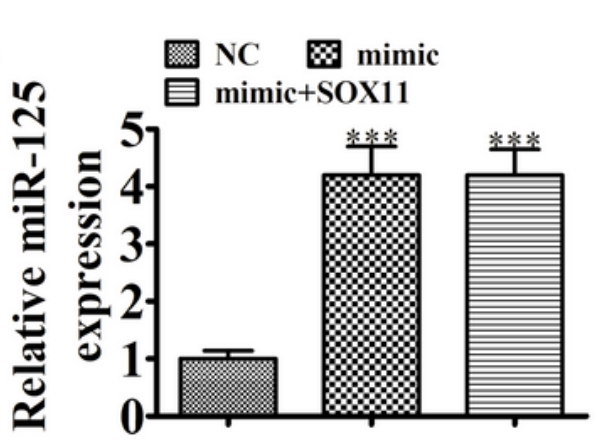

B

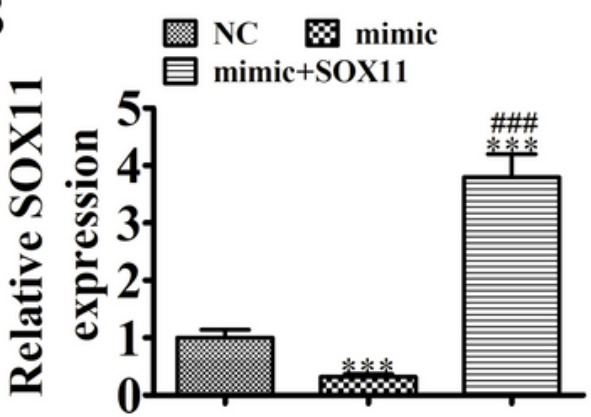

C
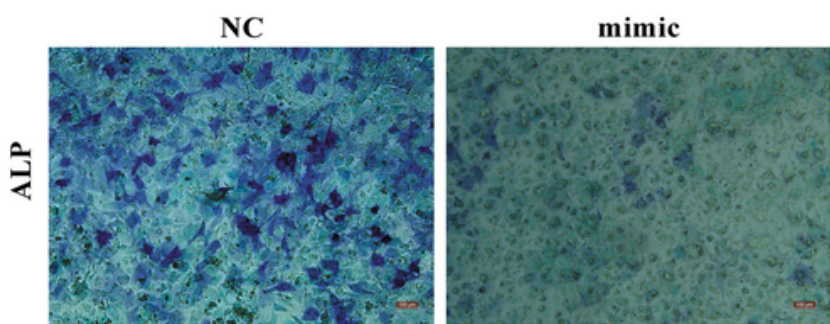

mimic+SOX11
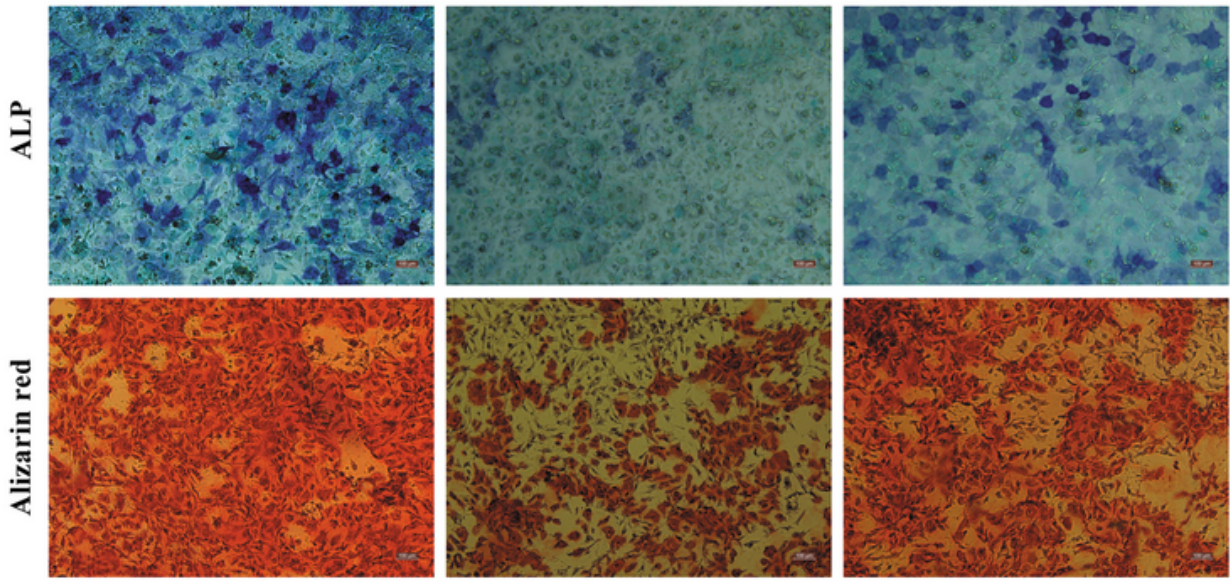

D

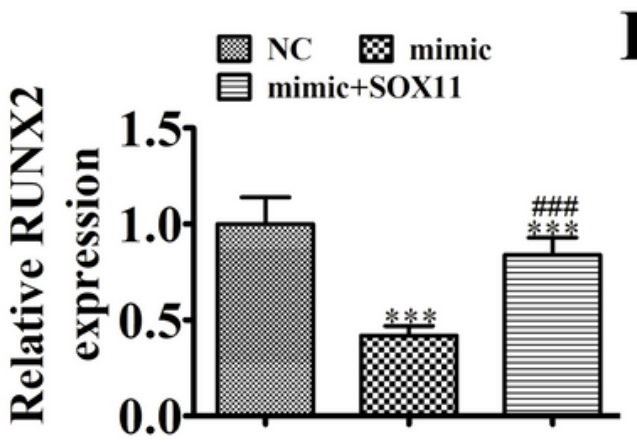

E

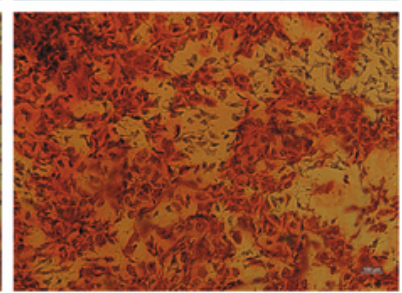

爻

0.0
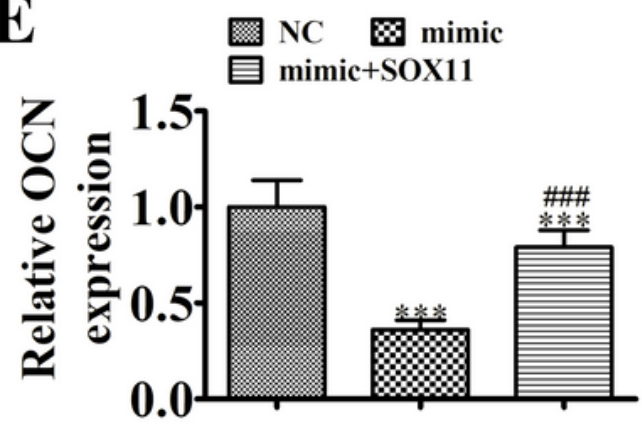

F

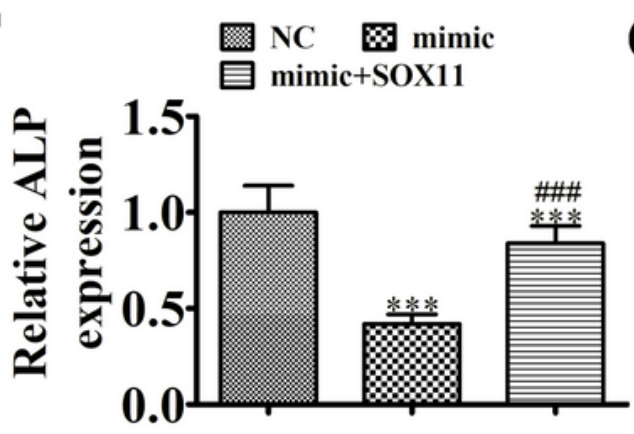

G
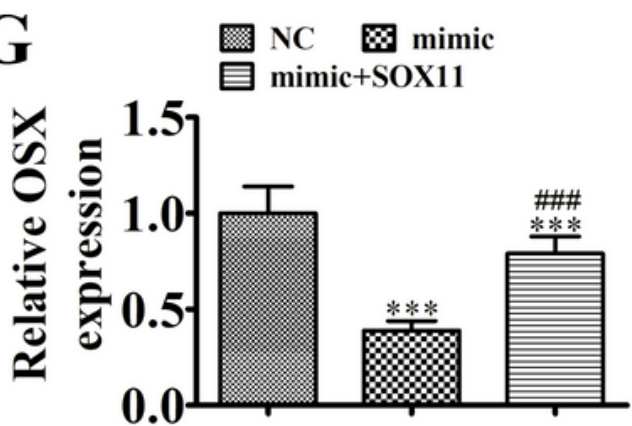

Figure 6

SOX11 overexpression restores miR-125 inhibition effect in osteogenic DPSC differentiation. (A-B) RTqPCR shows miR-125 and SOX11 expression. Data are presented as means \pm SD. ${ }^{\star *} * \mathrm{P}<0.001$ vs. NC. \#\#\#P<0.001 vs. mimic. (C) ALP images and Alizarin Red staining show osteogenic DPSC differentiation potential after induction for 14 days. (D-G) RT-qPCR shows RUNX2, OCN, ALP, and OSX expression. Results are presented as mean $\pm S D$. ${ }^{* *}{ }^{*} P<0.001$ vs. NC. \#\#\#P<0.001 vs. mimic. 
H\&E
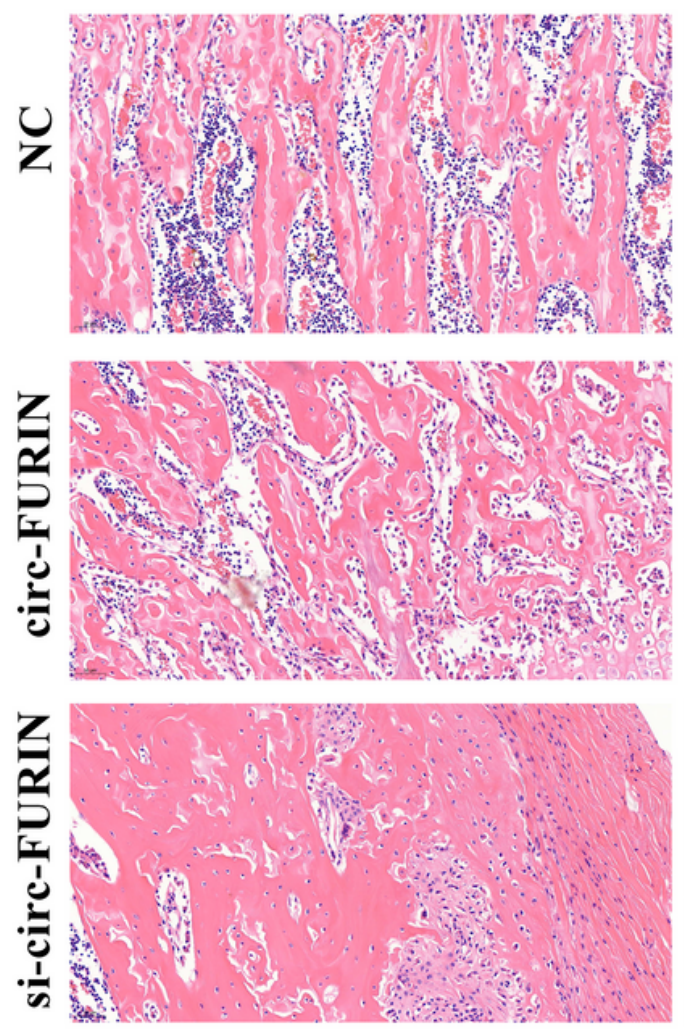

Masson
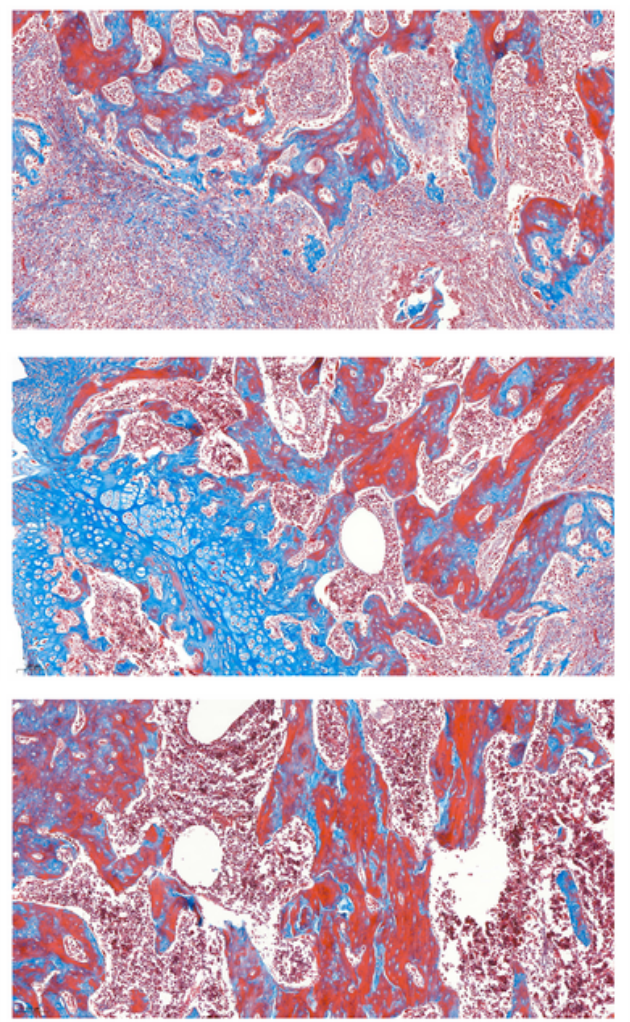

OCN
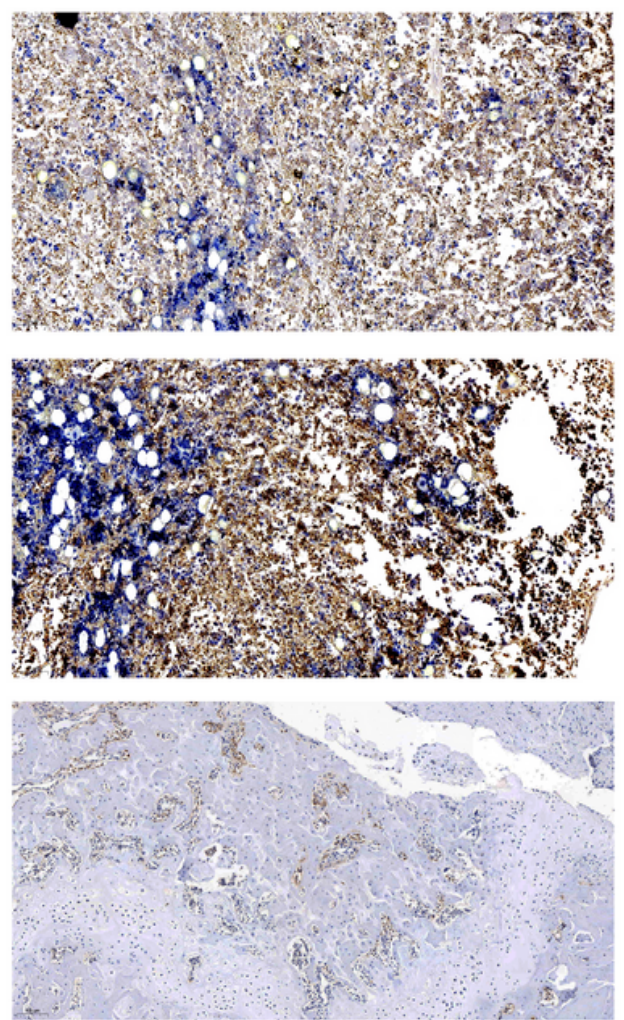

Figure 7

Circ-FURIN expression plays an important role in promoting heterotopic bone formation in vivo. H\&E, Masson's trichrome, and immunohistochemical staining of OCN in heterotopic bone. 